\title{
Beneficios educativos y videojuegos: revisión de la literatura española
}

\section{Educational Benefits of Videogames: Review of Spanish Journals}

\author{
Mario Grande de Prado \\ Universidad de León, León, España \\ mgrap@unileon.es \\ http://orcid.org/0000-0002-7180-8301
}

\section{Resumen}

El presente estudio aborda la revisión bibliográfica realizada sobre artículos en español (y editados en España) dentro del ámbito educativo sobre los videojuegos, y especialmente en sus beneficios. El periodo de tiempo analizado abarca los años 20132017, ambos incluidos. Los beneficios encontrados en esta consulta comprenden diversas perspectivas: desarrollar la competencia digital, nuevas propuestas educativas, su uso en colectivos como los alumnos con necesidades educativas especiales, mejora en diferentes aptitudes... Muchos de los artículos inciden en la motivación como una ventaja importante del uso de los videojuegos, destacando también el estímulo de las inteligencias múltiples y la creatividad. Los videojuegos comerciales más citados en la bibliografía consultada son los pertenecientes a la saga Assasin's Creed, el juego del rol online multijugador World of Warcraft y el juego creativo Minecraft, un sandbox con casi infinitas posibilidades.

\section{Palabras clave}

TIC; Videojuegos; Competencia digital; Nuevas propuestas educativas; Necesidades educativas especiales; Motivación

\begin{abstract}
The present study is a bibliographic review carried out in Spain with articles in Spanish within the educational field on videogames, and focused in their benefits. The lapse of time analyzed covers the period between 2013 and 2017, both included. The benefits found in this research include different perspectives: improving digital literacy, new educational proposals, its use in different groups, such as students with educational special needs, development of different skills... Many of the articles consider motivation as an important advantage of videogames use, also highlighting the stimulation of multiple intelligences and creativity. The commercial videogames most cited in the consulted bibliography are those belonging to the Assasin's Creed saga, the multiplayer online role-playing game (MMORPG) World of Warcraft and the creative game Minecraft, a sandbox with almost infinite possibilities.
\end{abstract}

\section{Keywords}

ICT; Videogames; Digital literacy; New educational proposals; Educational special needs; Motivation

\section{Introduction}

Los videojuegos pueden entenderse como juegos electrónicos con finalidad lúdica y un cierto grado de dificultad (Marcano, 2008). Básicamente, emplean la informática para permitir la interacción y retroalimentación inmediatos del jugador con la máquina, habitualmente mediante un soporte audiovisual (Tejeiro y Del Río, 2008). Se caracterizan (Gros, 2008), por la integración multimedia, la interactividad y su potencial educativo, además del enorme impacto en el ocio (Lorca, Cuenca, Vázquez y Velo, 2017). 
Aunque no estamos ante un recurso generalizado (Sánchez y Esnaola, 2014) -tal vez en parte debido a diferentes dificultades como las carencias en la formación, recursos disponibles y el interés y esfuerzo requerido para poner en marcha un proyecto (Cebrián, 2005; Del Moral y Fernández, 2015), o a los falsos mitos que los rodean (Grande, 2017)-, sin embargo no se puede negar que despiertan el interés de numerosos investigadores (Alonso y Yuste, 2014; Gros, 2008; Guerra y Revuelta, 2015; Ortega y Pérez, 2009; Quesada y Tejedor, 2016; Sampedro y McMullin, 2015).

Los videojuegos todavía son un tema complejo y polémico desde la perspectiva educativa; el objetivo de esta revisión consiste en analizar cuáles son los beneficios que aparecen en la bibliografía científica y su impacto en la producción científica reciente. El primero de los objetivos tiene respuesta en este mismo apartado, y el segundo en el análisis de resultados.

Con la intención de clarificar los beneficios de los videojuegos en educación, esta revisión del estado de la cuestión toma como referencia los artículos en español de las revistas educativas españolas disponibles en Dialnet e indexadas en Latindex. El término buscado ha sido "videojuegos" y el periodo analizado abarca desde 2013 hasta 2017.

La información encontrada se ha categorizado en:

- Beneficios.

- $\quad$ Riesgos y Dificultades.

- $\quad$ Riesgos y Beneficios.

Entre los artículos que inciden en los beneficios del uso educativo de los videojuegos, encontramos principalmente aquellos que:

1. Muestran propuestas educativas y los beneficios observados (Area y González, 2015; Del Moral, Guzmán y Fernández, 2014; García y Raposo, 2013; López y Jerez, 2015; Martín, 2015; Ramos y Botella, 2016; Téllez e Iturriaga, 2014). Varios incluyen el desarrollo de la competencia digital (Aguilar, Rubio y Viñals, 2013; Muros, Aragón y Bustos, 2013).

2. Aplican estos recursos a la intervención en colectivos concretos, como los alumnos con necesidades educativas especiales (Méndez y Lacasa, 2015; Sampedro y McMullin, 2015; Sánchez et al., 2017).

3. Destacan el papel de los videojuegos en la motivación (García y Raposo, 2013; Pinel et al., 2016; Rico y Agudo, 2016; Sampedro y McMullin, 2015; Sánchez et al., 2017).

Existen más aportaciones, pero estas son las tres que aglutinan la mayor parte de beneficios 
encontrados.

Profundizando en los aprendizajes que estas herramientas nos pueden facilitar, resulta importante subrayar aquellos relacionados con la alfabetización digital, el acceso y desarrollo de la competencia digital, términos que se emplean en ocasiones como sinónimos (Esteve y Gisbert, 2013). Esta competencia se puede ver beneficiada por el uso de este tipo de software (Aguilar, Rubio y Viñals, 2013; Díaz y Yuste, 2014; Gros, 2008; Muros, Aragón y Bustos, 2013; Quesada y Tejedor, 2016). Mediante juegos de estrategia, se promueve el aprendizaje de resolución de problemas, y tratamiento de la información y el conocimiento (Sampedro y McMullin, 2015; Torres, Romero, Pérez y Björk, 2016), dimensión fundamental de la competencia digital (INTEF, 2017; Ferrari, 2013; Grande, Cañón y Cantón, 2016). Es interesante indicar que Quesada y Tejedor (2016) analizaron el famoso videojuego de rol multijugador online World of Warcraft (WoW), concluyendo que este tipo de juegos ofrecen un amplio potencial en el desarrollo de competencias digitales.

Tras la competencia digital, destaca el aumento de la motivación (Agudo y Rico, 2016; Area y González, 2015; Díaz y Yuste, 2014; García y Raposo, 2013; Martín, 2016; Sampedro y McMullin, 2015; Sánchez, Ruiz y Sánchez, 2016), aspecto que parece presentar una base neurológica (Fernández, 2015). La motivación sin duda se debe entre otros factores a la retroalimentación e interactividad que les caracteriza (López y Jerez, 2015).

Otros estudios señalan también avances positivos en capacidades cognitivas, como memoria, comprensión, resolución de problemas; y psicomotoras, como por ejemplo destreza visual, discriminación perceptiva, coordinación espacial, etc. (AEVI, 2016). En este sentido, Del Moral, Guzmán y Fernández (2014) observan efectos positivos sobre las inteligencias múltiples de alumnos de Primaria, concretamente en la inteligencia lógico-matemática y la viso-espacial en niñas y lingüística e interpersonal en niños. Estos resultados relativos a las inteligencias múltiples son avalados por numerosas investigaciones citadas dentro de dicho estudio. Sampedro y McMullin (2015) por su parte subrayan el desarrollo de la atención y la memoria, además de pautas de convivencia y resolución de conflictos, esto es, competencia social cívica y toma de decisiones (Zhao y Linaza, 2015). Esto facilita desarrollar actitudes, por ejemplo, mediante la empatía (Guerra y Revuelta, 2015). Otra capacidad que los videojuegos pueden fomentar es la creatividad (Agudo y Rico, 2016; Guerra y Revuelta, 2015; Méndez y Del Moral, 2015; Méndez y Lacasa, 2015; Ortega y Pérez, 2009). En este sentido Guerra y Revuelta (2015b) emplearon un videojuego popular cuyas aplicaciones educativas son muy amplias: Minecraft, un entorno de creación prácticamente ilimitada donde se permite crear mundos e interaccionar en ellos. En este caso lo emplearon para trabajar emociones positivas con niños hospitalizados, mientras que otras investigaciones lo han empleado con adultos, encontrando también resultados positivos (Meier, Saorín, De la Torre, Bonnet y Melgar, 2016). Minecraft se emplea desde el año 2013 en las escuelas suecas (Gee, 2013; Holloway, 2013). 
Como podemos observar, muchos son los posibles beneficios del uso educativo de los videojuegos según la bibliografía consultada, destacando el desarrollo de la competencia digital, las capacidades cognitivas (atención, inteligencias múltiples, creatividad...), competencia social, la motivación...

\section{Metodología}

La metodología empleada es descriptivo-interpretativa, de revisión. Como referencia se toma la Revisión Sistemática de Literatura (SLR), aunque en nuestro caso la perspectiva empleada se acerca más a una visión global y exploratoria de la temática, propia del mapping. Se analizan los artículos publicados en España (y en español) en revistas científicas dentro del ámbito educativo.

La hemeroteca virtual Dialnet (www.dialnet.unirioja.es) nos muestra la existencia de 1.595 artículos hasta la fecha dentro de la búsqueda del término "videojuegos". De estos 1.013 ofrecen texto completo, 570 pertenecen a las materias de Psicología y Educación (353 específicamente a Educación) y 1097 han sido publicados desde 2010. Un total de 900 artículos han sido publicados en España, 1.014 en español, 700 están incluidos en Latindex y 280 emplean como descriptor "videojuegos".

Se han tomado como referencia las publicaciones con texto completo siguiendo como criterios de selección: artículos de revista completos, revistas indexadas en Latindex y publicadas en España (y en español) durante los años 2013-2017 y que contengan como palabra clave o descriptor "videojuegos".

Los artículos que cumplen dichos requisitos son treinta y cinco artículos, encontrándose la mayoría en bases de datos como CIRC 2012 o CARHUS+ (91,6\% y 68,6\%).

Cada artículo fue analizado mediante fichas sencillas y categorizado empleando posteriormente análisis estadísticos descriptivos. Además, se identificaron los principales beneficios.

\section{Análisis de resultados}

Tras el análisis de la revisión bibliográfica, se han organizado los resultados en base a:

Artículos por año de publicación.

Revistas consultadas.

Temática de los artículos analizados.

\subsection{Producción científica analizada por años}

Los resultados indican un incremento de la producción de literatura científica sobre este tema, destacando los años 2015 y 2016 como los años con mayor número de artículos (13 y 9 respectivamente), como se puede ver en la Figura 1 : 


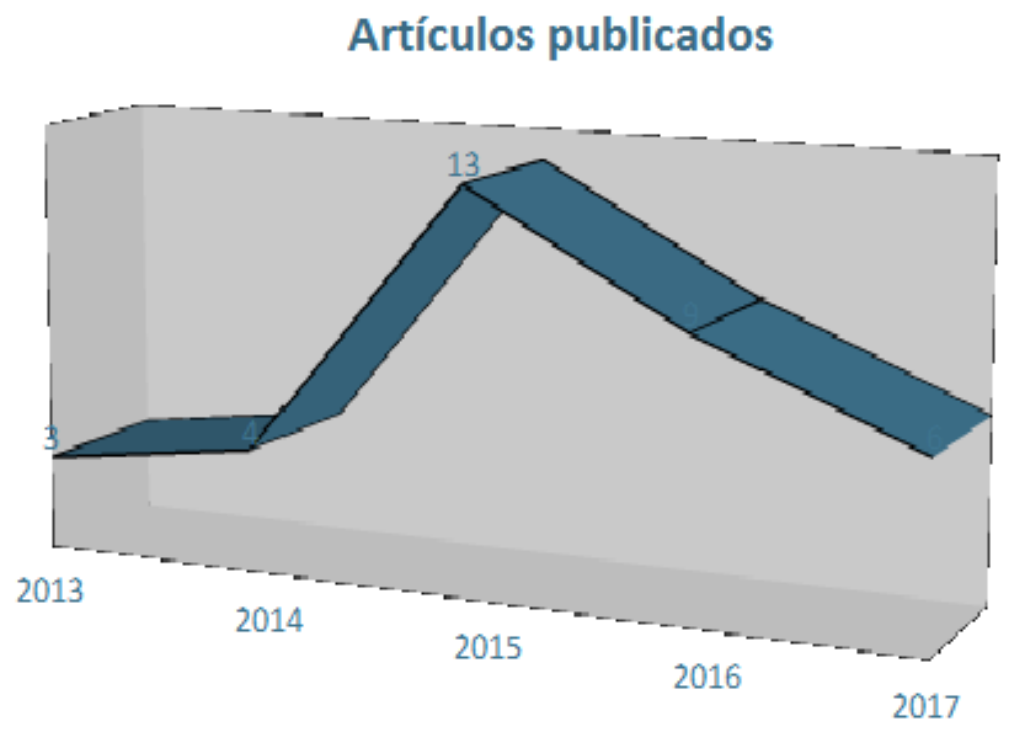

Figura 1. Publicaciones científicas educativas sobre videojuegos en España (2013 y 2017). Elaboración propia.

Esa tendencia parece atenuarse durante el 2017 (6 artículos), se mantiene por encima de la cantidad de artículos de los años 2013 y 2014 (3 y 4 respectivamente).

\begin{tabular}{|c|c|l|}
\hline Año & No. $^{\text {Autores }}$ \\
\hline 2013 & 3 & $\begin{array}{l}\text { Aguilar, Rubio y Viñals (2013); García y Raposo (2013); Muros, Aragón y } \\
\text { Bustos (2013). }\end{array}$ \\
\hline 2014 & 4 & $\begin{array}{l}\text { Alonso y Yuste (2014); Del Moral, Guzmán y Fernández (2014); Dezcallar, } \\
\text { Clariana, Cladelles, Badia y Gotzens (2014); Téllez e Iturriaga (2014). }\end{array}$ \\
\hline 2015 & 13 & $\begin{array}{l}\text { Ameneiros y Ricoy (2015); Area y Gonzálz (2015); Del Moral y Fernández } \\
\text { (2105); Del Moral, Fernández y Guzmán (2015); Espejo et al. (2015); Méndez } \\
\text { yacasa (2015); Guerra y Revuelta (2015); López y Jérez (2015); Martín } \\
\text { (2015); Méndez y Del Moral (2015); Navarrete y Molina (2015); Sampedro y } \\
\text { McMullin (2015); Zhao y Linaza (2015). }\end{array}$ \\
\hline 2017 & 9 & $\begin{array}{l}\text { Castillo, Meza, Garcés, y Camacho (2016); Cortés (2016); Guerra y Revuelta } \\
\text { (2016); Meier, Saorín, De la Torre, Bonnet y Melgar (2016); Pinel et al. (2016); } \\
\text { Rico y Agudo (2016); Ramos y Botella (2016); Ricoy y Ameneiros (2016); } \\
\text { Torres, Romero, Pérez y Björk (2016). }\end{array}$ \\
\hline $\begin{array}{l}\text { Correa, Duarte y Guzmán (2017); Chacón et al. (2017); Gómez, Planells y } \\
\text { Chicharro (2017); Lorca, Cuenca, Vázquez y Velo (2017); Sampedro, Muñoz } \\
\text { y Vega (2017); Sánchez, Ruiz y Sánchez (2017). }\end{array}$ \\
\hline
\end{tabular}

Tabla 1. Artículos científicos consultados clasificados por años de edición. Elaboración propia.

La Tabla 1 muestra los 35 artículos objeto de análisis, ordenados según el año de publicación. 


\subsection{Producción científica analizada por revistas}

Dentro de las revistas revisadas destacan RELATEC, Educar y Electronic Journal of Research in Educational Psychology (EJREP) con cuatro artículos cada una y Arte y Sociedad, Digital Education Review, Educatio Siglo XXI, Ocnos y Revista Complutense de Educación con dos publicaciones dentro de los años comprendidos entre 2013 y 2017 (Tabla 2).

Educar, EJREP y RELATEC aglutinan el 34,3\% de las publicaciones, respondiendo en el caso de las dos primeras a números especiales donde encontramos cuatro artículos en cada caso.

\begin{tabular}{|c|c|}
\hline Revistas & $\begin{array}{c}\text { Número de artículos } \\
\text { encontrados }\end{array}$ \\
\hline RELATEC; Educar; EJREP. & 4 \\
\hline $\begin{array}{c}\text { Arte y Sociedad; Digital Education Review; Educatio. } \\
\text { Siglo XXI; Ocnos; Revista Complutense de Educación. }\end{array}$ & 2 \\
\hline
\end{tabular}

Tabla 2. Revistas científicas con más de un artículo encontrado. Elaboración propia.

Otras revistas (un total de trece) han incluido un artículo en el período estudiado. Destaca Comunicar, ubicada en los primeros puestos JCR y SJCR, con siete artículos desde 2010, aunque dentro del periodo analizado solo aparece uno de ellos. También encontramos otras de importante trayectoria como Pixel Bit, Education in the Knowledge Society (EKS), Edutec, Qurriculum o Tendencias Educativas.

Otras revistas, muestran en este período varios artículos sobre aplicaciones concretas de un videojuego, no siendo incluidas en este estudio al emplear el descriptor "videojuego" y no "videojuegos".

\subsection{Temáticas predominantes en los artículos}

Sobre los enfoques abordados y la categorización de los documentos analizados, se ha optado por categorizar tomando como referencia fundamental el eje beneficios / riesgos.

De esta manera se han establecido tres categorías (Tabla 3), dos principales y dicotómicas -beneficios por un lado y riesgos y dificultades por otro-, completándolo con otra categoría más difusa en la que aparecen tanto unos como otros sin predominio claro de ninguno. 


\begin{tabular}{|l|c|}
\hline Categoría / Temática & Porcentaje de artículos \\
\hline Beneficios & $68,6 \%$ \\
\hline Riesgos y dificultades & $22,8 \%$ \\
\hline Riesgos y Beneficios & $8,6 \%$ \\
\hline
\end{tabular}

Tabla 3. Porcentajes de artículos científicos según la temática predominante. Elaboración propia.

Los beneficios son claramente mayoritarios en los artículos consultados (68,6\%), aunque podemos señalar que los riesgos alcanzan casi un tercio, si contabilizamos conjuntamente los artículos que enfatizan los riesgos (22,8\%) junto a aquellos que ponderan riesgos y beneficios (8,6\%).

A continuación, en las tablas 4, 5 y 6, aparecen resaltados los aspectos más relevantes dentro de cada categoría. Es importante señalar que algunos artículos se han contabilizado dentro de una misma categoría más de una vez según los aspectos en los que hayan incidido.

La Tabla 4 analiza los grandes bloques de beneficios encontrados.

\begin{tabular}{|c|c|c|}
\hline Beneficios destacados & Autores & $\begin{array}{l}\text { \% en esta } \\
\text { categoría }\end{array}$ \\
\hline $\begin{array}{l}\text { Favorecer inteligencias múltiples } \\
\text { / competencias / capacidades } \\
\text { / habilidades (destacando } \\
\text { metacognitivas, sociales, } \\
\text { autonomía, creatividad y } \\
\text { competencia digital) }\end{array}$ & $\begin{array}{l}\text { Aguilar et al. (2013); Del Moral et al. (2015); Del } \\
\text { Moral et al. (2014); García y Raposo (2013); Guerra } \\
\text { y Revuelta (2015); Meier et al. (2016); Méndez y } \\
\text { Del Moral (2015); Muros et al. (2013); Rico y Agudo } \\
\text { (2016); Sampedro y McMullin (2015); Sampedro et al. } \\
\text { (2017); Sánchez et al. (2017); Zhao y Linaza (2015). }\end{array}$ & $54,2 \%$ \\
\hline Propuesta / experiencia & $\begin{array}{l}\text { Castillo et al. (2016); Del Moral et al. (2014); García } \\
\text { y Raposo (2013); Guerra y Revuelta (2015); López y } \\
\text { Jérez (2015); Martín (2015); Ramos y Botella (2016); } \\
\text { Rico y Agudo (2016); Sampedro et al. (2017); Téllez e } \\
\text { Iturriaga (2014). }\end{array}$ & $41,2 \%$ \\
\hline Motivación & $\begin{array}{l}\text { Alonso y Yuste (2014); García y Raposo (2013); Pinel } \\
\text { et al. (2016); Rico y Agudo (2016); Sampedro et al. } \\
\text { (2017); Sampedro y McMullin (2015); Sánchez et al. } \\
\text { (2017). }\end{array}$ & $29,2 \%$ \\
\hline $\begin{array}{l}\text { Inclusión educativa / Atención a } \\
\text { la Diversidad }\end{array}$ & $\begin{array}{l}\text { Méndez y Lacasa (2015); Sampedro y McMullin } \\
\text { (2015); Sánchez et al. (2017). }\end{array}$ & $12,5 \%$ \\
\hline $\begin{array}{l}\text { Actitud favorable de los jóvenes } \\
\text { y futuros docentes }\end{array}$ & Correa et al. (2017); Lorca et al. (2017). & $8,3 \%$ \\
\hline $\begin{array}{l}\text { Videojuegos y materiales } \\
\text { pedagógicos }\end{array}$ & Area y González (2015). & $4,2 \%$ \\
\hline
\end{tabular}


Dentro de los beneficios, destaca el desarrollo de inteligencias múltiples (o competencias / capacidades, entre las que se incluyen: metacognición, autonomía, competencia social, competencia digital autonomía y creatividad) (54,2\%), varias propuestas y experiencias concretas $(41,2 \%)$, la motivación $(12,5 \%)$ y la actitud de futuros docentes (8,3\%), que podemos considerar un apartado dentro de la motivación (del profesorado, en este caso).

La Tabla 5 profundiza en los riesgos y dificultades.

\begin{tabular}{|l|l|c|}
\hline \multicolumn{1}{|c|}{$\begin{array}{c}\text { Riesgos y } \\
\text { dificultades } \\
\text { destacados }\end{array}$} & \multicolumn{1}{|c|}{ Autores } & $\begin{array}{c}\text { \% en esta } \\
\text { categoría }\end{array}$ \\
\hline Adicción & Chacón et al. (2017); Espejo et al. (2016); Ricoy y Ameneiros (2016). & $60 \%$ \\
\hline $\begin{array}{l}\text { Estereotipos } \\
\text { sexistas }\end{array}$ & Cortés (2016); Guerra y Revuelta (2016). & $42,9 \%$ \\
\hline $\begin{array}{l}\text { Desconocimiento } \\
\text { de adultos }\end{array}$ & Del Moral y Fernández (2105); Guerra y Revuelta (2016). & $28,6 \%$ \\
\hline Violencia & Navarrete y Molina (2015). & $14,3 \%$ \\
\hline $\begin{array}{l}\text { Menor tiempo de } \\
\text { lectura y estudio }\end{array}$ & Dezcallar et al. (2014). & $14,3 \%$ \\
\hline
\end{tabular}

Tabla 5. Artículos científicos sobre riesgos y dificultades de los videojuegos. Elaboración propia.

Sobre los riesgos, la adicción (60\%), los estereotipos (42,9\%) y el desconocimiento de progenitores y docentes $(28,6 \%)$ destacan especialmente, estando este último relacionando tanto con la violencia $(14,3 \%)$ como con los estereotipos (42,9\%).

Para terminar el análisis por categorías, la Tabla 6 ofrece los artículos que no se incluyen claramente en ninguna de las dos categorías principales.

\begin{tabular}{|l|l|l|}
\hline \multicolumn{1}{|c|}{ Beneficios destacados } & \multicolumn{1}{c|}{ Riesgos y dificultades destacados } & \multicolumn{1}{c|}{ Autores } \\
\hline $\begin{array}{l}\text { Actitud positiva de los } \\
\text { jóvenes }\end{array}$ & Estereotipos sexistas & Gómez et al. (2017) \\
\hline $\begin{array}{l}\text { Favorecer múltiples } \\
\text { capacidades }\end{array}$ & Violencia & $\begin{array}{l}\text { Ameneiros y Ricoy } \\
(2015)\end{array}$ \\
\hline $\begin{array}{l}\text { Salud mental. Competencia } \\
\text { social }\end{array}$ & Adicción & Torres et al. (2016) \\
\hline
\end{tabular}

Tabla 6. Artículos científicos sobre riesgos y beneficios de los videojuegos. Elaboración propia. 
Estos artículos (un 8,6\% del total), vienen a subrayar en los beneficios las capacidades y competencias, así como la actitud de los jóvenes (motivación). Sobre los riesgos, inciden en los dos riesgos principales detectados (adicción y estereotipos), y la violencia.

\section{Discusión y conclusiones}

La evolución tecnológica proporciona nuevos instrumentos para el ocio, que poseen un enorme potencial en su uso educativo (Fernández, 2015).

Algunos autores ponen el énfasis en sus posibles beneficios (Agudo y Rico, 2015; Chacon et al., 2017; Del Moral, Fernández y Guzmán, 2015; Espejo et al., 2016; Quesada y Tejedor, 2016), mientras que otros señalan los riesgos (Guerra y Revuelta, 2016; Ricoy y Ameneiros, 2015; 2016). La clave probablemente reside en cómo son usados (López y Jerez, 2015).

La producción de artículos muestra una mayor cantidad de artículos en los últimos años (2015-2017), siendo el 2015 el que aporta más publicaciones, seguido por 2016 y 2017 con resultados algo más discretos.

Sobre las revistas que incluyen los artículos analizados, muchas tratan este tema (un total de 21), aunque con un contacto más bien esporádico en este periodo de tiempo, incluyendo la mayoría (13 de 21) un único artículo en el quinquenio 2013-2018.

Desde el punto de vista cuantitativo, predominan propuestas y estudios sobre sus beneficios (68,6\%), frente a los riesgos y dificultades $(31,4 \%)$, incluyendo dentro de ese porcentaje aquellos artículos que citan tanto beneficios como riesgos sin un predominio evidente $(8,6 \%)$.

Dentro de los beneficios, en los artículos analizados se hayan el desarrollo de múltiples capacidades (incluyendo competencia digital y social, creatividad...), diversas propuestas y experiencias, la motivación y el potencial dentro de la Educación Inclusiva.

Sobre los riesgos, la adicción, los estereotipos y la violencia, potenciados por la brecha digital de los adultos responsables, parecen configurarse como el lado más oscuro de los videojuegos en la literatura educativa.

Este es, por tanto, el mapping que nos ofrecen los artículos consultados en español y editados en España entre los años 2013 y 2017, pudiendo resultar interesante la comparación de estos datos con otros países.

Para concluir, parece evidente que es una temática que genera interés en el ámbito educativo español pero que no parece contar, todavía al menos, con una presencia continuada en la mayor parte de 
las revistas. Sea como fuere, los videojuegos han llegado para quedarse y debemos considerar sus posibles riesgos pero también aprovechar el potencial que nos brindan. Contamos con la actitud favorable de jóvenes y educadores hacia el uso de videojuegos educativos (Gómez et al., 2016; Lorca et al., 2017) y, por ello, se debe prestar atención a la formación inicial y continua del profesorado, así como a la dotación de recursos, que juegan un papel fundamental en la implementación de las TIC (Cebrián, 2005; Del Moral y Fernández, 2015; Correa et al., 2017).

Si logramos afianzar y optimizar su uso, las investigaciones consultadas muestran prometedores avances en varios aspectos como, por ejemplo, el desarrollo de la competencia digital, aumento de la motivación y el estímulo de diferentes capacidades cognitivas (como la atención, las inteligencias múltiples y la creatividad). Convienen por tanto profundizar en dichas aportaciones que abarcan parcelas del desarrollo integral y del aprendizaje que son complejas de abordar, encontrándonos ante un reto que ofrece un potencial que no puede ser desdeñado por los educadores.

\section{Referencias}

AEVI (Asociación Española de Videojuegos) (2016). Anuario de la industria de Videojuegos. Recuperado de: https://goo.gl/JCTsaM

Aguilar Gutiérrez, E., Rubio Florido, I. y Viñals Blanco, A. (2013). El ocio digital como recurso para el aprendizaje, la socialización y la generación de capital social. Revista de la Asociación de Sociología de la Educación (RASE), 6(2), 196-209

Alonso Díaz, L. y Yuste Tosina, R. (2015). Teorías de la educación de adultos que subyacen en el uso de videojuegos. Education in the Knowledge Society (EKS), 15(4), 160-183.

Ameneiros, A. y Ricoy Lorenzo, M.C. (2015). Los videojuegos en la adolescencia: prácticas y polémicas asociadas. Revista de Estudios e Investigación en Psicología y Educación, 13, 115119. doi:https://doi.org/10.17979/reipe.2015.0.13.451

Area Moreira, M. y González González, C. (2015). De la enseñanza con libros de texto al aprendizaje en espacios online gamificados. Educatio Siglo XXI, 33(3), 15-38. doi: https://doi. org/10.6018/j/240791

Castillo, E., Meza, E., Garcés, S. y Camacho, W. (2016). Micromundo etnoeducativo como apoyo al docente en las prácticas de enseñanza de la lengua nam trik de Totoró: "namoipo jaumai amkun”. En Computing Conference (CCC), 2016 IEEE 11th Colombian (pp. 1-8). EEUU: IEEE. doi: https://doi.org/10.1109/ColumbianCC.2016.7750775

Cañón, R., Grande, M. y Cantón, I. (2016). Brecha digital: Impacto en el desarrollo social y 
personal. Factores asociados. Tendencias Pedagógicas, 28, 115-132. doi: https://doi. org/10.15366/tp2016.28.009

Cebrián de la Serna, M. (2005). Tecnologías de la Información y Comunicación para la formación de docentes. Madrid: Pirámide.

Correa García, R., Duarte Hueros, A. y Guzmán Franco, M. (2017). Horizontes educativos de los videojuegos. Propuestas y reflexiones de futuros maestros y educadores sociales. Educar, 53(1), 67-88. doi: https://doi.org/10.5565/rev/educar.849

Cortés-Picazo, L. (2016). Transgresión de las identidades tradicionales de género mediante la representación gráfica de mujeres protagonistas para videojuegos desarrollada por niños y niñas. Arte, Individuo Y Sociedad, 28(3), 459-473. doi: https://doi.org/10.5209/rev_ARIS.2016. v28.n3.48951

Chacón Cuberos, R., Zurita Ortega, F., Martínez Martínez, A., Castro Sánchez, M., Espejo Garcés, T. y Pinel Martínez, C. (2017). Relación entre factores académicos y consumo de videojuegos en universitarios. Un modelo de regresión. Píxel-Bit. Revista de Medios y Educación, 50, 109121. doi: https://doi.org/10.12795/pixelbit

Del Moral Pérez, M. E. y Fernández García, L. C. (2015). Videojuegos en las aulas: implicaciones de una innovación disruptiva para desarrollar las Inteligencias Múltiples. Revista Complutense de Educación, 26(Nº. Extra 1), 97-118. doi: https://doi.org/10.5209/rev_RCED.2015.v26.44763

Del Moral Pérez, M. E., Fernández García, L. C. y Guzmán Duque, A. P. (2015). Videojuegos: incentivos multisensoriales potenciadores de las inteligencias múltiples en Educación Primaria. Electronic Journal of Research in Educational Psychology, 13(36), 243-270. doi: https://doi.org/10.25115/ejrep.36.14091

Del Moral Pérez, M., Guzmán Duque, A. y Fernández, L. (2014). Serious Games: escenarios lúdicos para el desarrollo de las inteligencias múltiples en escolares de primaria. Edutec, 47. doi: https://doi.org/10.21556/edutec.2014.47.121

Dezcallar, T., Clariana, M., Cladelles, R., Badia, M. y Gotzens, C. (2014). La lectura por placer: su incidencia en el rendimiento académico, las horas de televisión y las horas de videojuegos. Ocnos, 12, 107-116. doi: https://doi.org/10.18239/ocnos_2014.12.05

Díez, E. J. (2014). Video games and gender-based violence. Procedia-Social and Behavioral Sciences, 132, 58-64. doi: https://doi.org/10.1016/j.sbspro.2014.04.278

Espejo Garcés, T., Chacón Cuberos, R., Castro Sánchez, M., Martínez Martínez, A., Zurita 
Ortega, F. y Pinel Martínez, C. (2015). Análisis descriptivo del uso problemático y hábitos de consumo de los videojuegos con relación al género en estudiantes universitarios. RELATEC: Revista Latinoamericana de Tecnología Educativa, 14(3), 85-93

Fernández, I. (2015). Juego serio: gamificación y aprendizaje. Comunicación y pedagogía: Nuevas tecnologías y recursos didácticos, 281, 43-48.

Ferrari, A. (2013). DIGCOMP: A Framework for Developing and Understanding Digital Competence in Europe. (Y. Punie y N. Barbara, eds.). Luxemburgo: Publications Office of the European Union. Recuperado de https://goo.gl/4pa1Sd

García Rodríguez, M.F. y Raposo Rivas, M. (2013). Trabajando con videojuegos en el aula: una experiencia con Wii Music. Tendencias pedagógicas, 22, 45-58.

Gee, O. (2013). Swedish school makes Minecraft a must. The Local, 9.

Gómez-García, S., Planells de la Maza, A., y Chicharro-Merayo, M. (2017). ¿Los alumnos quieren aprender con videojuegos? Lo que opinan sus usuarios del potencial educativo de este medio. Educar, 53(1), 49-66. doi: https://doi.org/10.5565/rev/educar.848

Grande de Prado, M., Cañón, R. y Cantón, I. (2016). Competencia digital y tratamiento de la información en futuros maestros de primaria. En Educatio Siglo XXI, 34(3), 101-118. doi: https://doi.org/10.6018/j/275961

Grande de Prado, M. (2017). Videojuegos y alfabetización digital: mitos y rechazo. En López Meneses, E., Cobos Sanchiz, D., Martin Padilla, A. H., Molina García, L. \& Jaén Martínez, A. (Eds). INNOVAGOGÍA 2016: III Congreso Internacional Virtual innovación pedagógica y praxis educativa. Libro de Actas: 28, 29 y 30 de noviembre de 2016. Congreso Virtual Internacional sobre Innovación Pedagógica y Praxis Educativa. AFOE Formación: Sevilla (España).

Gros, B. (2008). Videojuegos y aprendizaje. Barcelona: Grao.

Guerra Antequera, J. y Revuelta Domínguez, F. I. (2015a). Visión y tratamiento educativo de los roles masculino y femenino desde el punto de vista de los videojugadores: tecnologías emergentes favorecedoras de la igualdad de género. Qurriculum: Revista de teoría, investigación y práctica educativa, 28, 142-160.

Guerra Antequera, J. y Revuelta Domínguez, F. I. (2015b). Videojuegos precursores de emociones positivas: propuesta metodológica con Minecraft en el aula hospitalaria. IJERI: International Journal of Educational Research and Innovation, 3, 105-120. 
Guerra Antequera, J. y Revuelta Domínguez, F. I. (2016). Análisis del conocimiento del código PEGI en la formación inicial del profesorado. RELATEC: Revista Latinoamericana de Tecnología Educativa, 15(1), 87-96.

Holloway, J. (2013, January 14). School imposes compulsory Minecraft lessons [Blog]. Recuperado de https://goo.gl/VbfFFM

INTEF (2017). Marco Común de Competencia Digital Docente del Plan de Cultura Digital en la Escuela. MECD. Recuperado de: https://goo.gl/JjpFqs

López Valero, A. y Jerez Martínez, I. (2015). Textualidad digital y multialfabetización. Los contenidos digitales como material educativo. Educatio siglo XXI: Revista de la Facultad de Educación, 33(2), 165-182. doi: https://doi.org/10.6018/j/233191

Lorca Marín, A. A., Cuenca López, J. M., Vázquez Bernalo, B. y Velo, S. (2017). Actitudes de los docentes en formación inicial sobre videojuegos. Digital Education Review, 31, 39-60.

Marcano, B. (2008). Juegos serios y entrenamiento en la sociedad digital. Education in Knoledge Society (EKS), 9(3), 93-107.

Martín Rodríguez, I. (2016). Don Quijote, Player 1. El Guiniguada. Revista de investigaciones y experiencias en Ciencias de la Educación, 24, 27-31.

Meier, C., Saorín Pérez, J. L., De la Torre Cantero, J., Bonnet de León, A. y Melgar Ramírez, M. (2016). Construcción de un mundo virtual en Minecraft para el aprendizaje del patrimonio escultórico urbano. RELATEC: Revista Latinoamericana de Tecnología Educativa, 15(3), 69-81.

Méndez Zaballos, L. y Del Moral Pérez, M.E. (2015). Presentación: Investigación e innovación educativa con videojuegos. Electronic Journal of Research in Educational Psychology, 13(36), 211-218. doi: https://doi.org/10.14204/ejrep.36.15079

Méndez Zaballos, L. y Lacasa Díaz, P. (2015). Los videojuegos, herramientas para el cambio: un estudio desde la teoría de la actividad. Electronic Journal of Research in Educational Psychology, 13(36), 271-300. doi: https://doi.org/10.14204/ejrep.36.14099

Muros Ruiz, B., Aragón Carretero, Y. y Bustos Jiménez, A. (2013). La ocupación del tiempo libre de jóvenes en el uso de videojuegos y redes. Comunicar, 20(40), 31-39. doi: https://doi. org/10.3916/C40-2013-02-03

Navarrete-Cardero, L. y Molina-González, J. (2015). La influencia de los videojuegos de 
contenido apocalíptico en los adolescentes. Arte, Individuo y Sociedad, 27(2), 161-178. doi: https://doi.org/10.5209/rev_ARIS.2015.v27.n2.43176

Pérez, Á. y Ortega, J. A. (2009). El potencial didáctico de los videojuegos: "The Movies" un videojuego que fomenta la creatividad audiovisual.Etic@net, 9(10), 2.

Pinel Martínez, C., Zurita Ortega, F., Espejo Garcés, T., Chacón Cuberos, R., Castro Sánchez, M. y Pérez Cortés, A. J. (2016). La interacción de la frecuencia de juego con la adquisición de hábitos saludables en escolares de Granada. RELATEC: Revista Latinoamericana de Tecnología Educativa, 15(2), 169-176.

Ramos Ahijado, S. y Botella Nicolás, A. M. (2016). Los videojuegos como herramientas de aprendizaje: una experiencia de innovación con la ópera de Mozart. Dedica. Revista de Educação e Humanidades, 9, 161-171.

Rico-García, M. y Agudo Garzón, J. (2016). Aprendizaje móvil de inglés mediante juegos de espías en educación secundaria. RIED. Revista Iberoamericana de Educación a Distancia, 19(1), 121-139. doi: https://doi.org/10.5944/ried.19.1.14893

Ricoy, C., y Ameneiros, A. (2016). Preferencias, dedicación y problemáticas generadas por Ios videojuegos: una perspectiva de género. Revista Complutense De Educación, 27(3), 1.2911.308. doi: https://doi.org/10.5209/rev_RCED.2016.v27.n3.48445

Roig, R., Mengual, S. y Quinto, P. (2015). Conocimientos tecnológicos, pedagógicos y disciplinares del profesorado de Primaria. Comunicar, 23(45) 151-159. doi: https://doi. org/10.3916/C45-2015-16

Sampedro Requena, B. y McMullin, K. J. (2015). Videojuegos para la inclusión educativa. Digital Education Review, 27, 122-137.

Sampedro Requena, B., Muñoz González, J. y Vega Gea, E. (2017). El videojuego digital como mediador del aprendizaje en la etapa de Educación Infantil. Educar, 53(1), 89-107. doi: https:// doi.org/10.5565/rev/educar.850

Sánchez Peris, F.J. y Esnaola Horacek, G.A. (2014). Aularia: Revista Digital de Comunicación, 3(1), 21-26.

Sánchez-Rivas, E., Ruiz-Palmero, J. y Sánchez-Rodríguez, J. (2017). Videojuegos frente a fichas impresas en la intervención didáctica con alumnado con necesidades educativas especiales. Educar, 53(1), 29-48. doi: https://doi.org/10.5565/rev/educar.844 
Téllez Alarcia, D. y Iturriaga Barco, D. (2014). Videojuegos y aprendizaje de la Historia: la saga Assasin's Creed. Contextos educativos: Revista de Educación, 17, 145-155. doi: https://doi. org/10.18172/con.2598

Torres-Toukoumidis, A., Romero-Rodríguez, L., Pérez-Rodríguez, M. A. y Björk, S. (2016). Desarrollo de habilidades de lectura a través de los videojuegos: estado del arte. Ocnos, 15 (2), 37-49. doi: https://doi.org/10.18239/ocnos_2016.15.2.1124

Zhao, Z. y Linaza Iglesias, J. L. (2015). La importancia de los videojuegos en el aprendizaje y el desarrollo de niños de temprana edad. Electronic Journal of Research in Educational Psychology, 13(36), 301-318. doi: https://doi.org/10.14204/ejrep.36.14108 